\title{
On the Zero Divisor Graphs of a Class of Commutative Completely Primary Finite Rings \\ Walwenda Shadrack Adero ${ }^{1}$ and Owino Maurice Oduor ${ }^{2}$ \\ 1 Maseno University, Maseno, KENYA \\ awalwenda@yahoo.com \\ 2 University of Kabianga, Kericho, KENYA \\ moduor@kabianga.ac.ke
}

\section{Abstract}

Let $R$ be a Completely Primary Finite Ring with a unique maximal ideal $Z(R))$, satisfying $\left((Z(R))^{n-1} \neq(0)\right.$ and $(Z(R))^{n}=(0)$. The structures of the units some classes of such rings have been determined. In this paper, we investigate the structures of the zero divisors of $R$.

Keywords: Finite rings; Zero divisor graphs.

Classification (MSC2010): 05C25, 05C35.

\section{Introduction}

It is well known, that in a finite ring with 1 , an element is either a zero divisor or a unit. Let $R$ be a finite ring with 1 . An element $u \in R$ is called a zero divisor, if there exists some nonzero element $v \in R$, such that $u v=0$. The set of zero divisors in $R$ is denoted by $Z(R)$. The zero divisor graphs considered in this paper are due to Anderson and Livingstone in [3], denoted by $\Gamma(R)$. It is conventional, that these graphs present a better description of the structures of the zero divisors. The rest of the notations to be used in the sequel can be obtained from $[1,2,4,5]$.

Galois rings are completely primary and the importance of completely primary rings in the structure theory of finite rings cannot be underestimated. This is due to the fact that, a commutative finite ring with identity is expressible as a direct sum of completely primary finite rings. The building blocks for the construction of completely primary finite rings are the Galois rings, which are basically the extensions of rings of integers modulo $p^{n}$, where $p$ is a prime integer and $n$ is a positive integer. In the next section, we give the construction of the ring under consideration.

\section{The Construction}

Let $R^{\prime}$ be the Galois ring of the form $G R\left(p^{k r}, p^{k}\right)$. For each $i=1, \cdots, h$, let

$u_{i} \in Z(R)$ and $U$ be an $h$-dimensional $R^{\prime}$-module generated by $\left\{u_{1}, \cdots, u_{h}\right\}$ such that $R=R^{\prime} \oplus U$ is an additive group. On this group, define multiplication by the following relations;

$$
\begin{aligned}
& \text { - If } k=1,2 \text {, then } p u_{i}=u_{i} u_{j}=u_{j} u_{i}=0, u_{i} r^{\prime}=\left(r^{\prime}\right)^{\sigma_{i}} u_{i} \text {. } \\
& \text { - If } k \geq 3 \text {, then } p^{k-1} u_{i}=0, u_{i} u_{j}=p^{2} \gamma_{i j}, u_{i}^{k}=u_{i}^{k-1} u_{j}=u_{i} u_{j}^{k-1}=0 ;
\end{aligned}
$$

$u_{i} r^{\prime}=\left(r^{\prime}\right)^{\sigma_{i}} u_{i}$, where $r^{\prime}, \gamma_{i j} \in R^{\prime}, 1 \leq i, j \leq h, p$ is a prime integer, $k$ and $r$ are positive integers and $\sigma_{i}$ is the automorphism associated with $u_{i}$. Further, let the generators $\left\{u_{i}\right\}$ for $U$ satisfy the additional condition that if $u_{i} \in U$, then $p u_{i}=u_{i} u_{j}=0$.

From the given construction, we notice that $R$ is commutative iff $\sigma_{i}$ is an identity automorphism. The ring is also completely primary and the subset of the zero divisors $Z(R)$ satisfies the following: $Z(R)=p R^{\prime} \oplus U$; $(Z(R))^{n-1}=p^{n-1} R^{\prime}$ and $(Z(R))^{n}=(0)$.

Due to the differences in their geometrical and structural properties, we present the results of the graph $\Gamma(R)$ for each characteristic of $R$. 


\section{Rings of characteristic $p$}

Proposition 3.1 Let char $R=p$. Then

i) $|\Gamma(R)|=p^{r h}-1$

ii) $\Gamma(R)$ is complete

iii) $\Gamma(R)=K_{p^{r h}}$.

iv) $\operatorname{diam}(\Gamma(R))=1$.

v) $\operatorname{gr}(\Gamma(R))=\{\infty$ if $r=1, h=0,1$ and $p=2,3$.

$\{3$ elsewhere.

vi) The binding number $b(\Gamma(R))=\infty$.

\section{Proof.}

i) By the above construction, we have that $R^{\prime}=G F\left(p^{r}\right)$ and $F=R^{\prime} / p R^{\prime}$. So $U=F^{h}$ is an $R^{\prime}$ -module generated by $u_{1}, \ldots, u_{h}$ on the additive group $R=R^{\prime} \oplus U$. It is clear that $Z(R)=R^{\prime} u_{1} \oplus R^{\prime} u_{2} \oplus, \ldots, \oplus R^{\prime} u_{h}$ and every non zero element in $Z(R)$ is of the form $\left(0, r_{1}, r_{2}, \ldots, r_{h}\right)$, for some $r_{i} \neq 0$. We show that any element not contained in $Z(R)$ is invertible. So let $\left(r_{0}, r_{1}, r_{2}, \ldots, r_{h}\right) \notin Z(R)$. Choose an element say $\left(s_{0}, s_{1}, s_{2}, \ldots, s_{h}\right) \notin Z(R)$ such that $\left(r_{0}, r_{1}, r_{2}, \ldots, r_{h}\right)\left(s_{0}, s_{1}, s_{2}, \ldots, s_{h}\right)=(1,0,0, \ldots, 0)$. This implies that $r_{0} s_{0}=1 s_{0}=r_{0}^{-1} \quad$ and $r_{0} s_{i}+r_{i} s_{0}=0 s_{i}=-r_{i} r_{0}^{-2}$ for $1<i \leq h$. So, $\left(r_{0}, r_{1}, r_{2}, \ldots, r_{h}\right)^{-1}=\left(r_{0}^{-1},-r_{1} r_{0}^{-2},-r_{2} r_{0}^{-2}, \ldots,-r_{h} r_{0}^{-2}\right)$. Since $|R|=\left|R^{\prime} \| U\right|=p^{(h+1) r}|Z(R)|=p^{r h}$ and $\Gamma(R)=Z(R)^{*}$, then $|\Gamma(R)|=p^{r h}-1$ which establishes (i).

ii) To establish this, note that the product of every pair $\left(0, r_{1}, r_{2}, \ldots, r_{h}\right)$, $\left(0, s_{1}, s_{2}, \ldots, s_{h}\right) \in Z(R)-\{0\}$ is equal to zero so that every pair of vertices in $\Gamma(R)$ are adjacent.

Hence $\Gamma(R)$ is complete.

iii) This is a consequence of (ii), since $\Gamma(R)$ is complete having $p^{r h}-1$ vertices.

iv) This follows from (ii).

v) When $r=1, h=0$ or 1 and $p=2$ or $3, n=\left(p^{r h}-1\right) \leq 2$. So $\operatorname{gr}(\Gamma(R))=\infty$. Otherwise, $n=\left(p^{r h}-1\right)>2$ and since the completeness of the graph implies that $\operatorname{gr}(\Gamma(R))=2 \operatorname{diam}(\Gamma(R))+1$ , the result readily follows.

vi) Since the set $S$ of minimal degree in $V(\Gamma(R))$ is empty, $b(\Gamma(R))=\infty$.

\section{Rings of characteristic $p^{2}$}

Proposition 4.1 Let char $R=p^{2}$. Then the following hold:

i) $|\Gamma(R)|=p^{(h+1) r}-1$

ii) $\Gamma(R)$ is complete.

iii) $\Gamma(R)=K_{p^{(h+1) r}-1}$

iv) $\operatorname{diam}((\Gamma(R)))=1$

v) $\operatorname{gr}(\Gamma(R))=\{\infty$ if $r=1, h=0$, and $p=2,3$ 


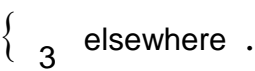

vi) The binding number $b(\Gamma(R))=\infty$.

\section{Proof.}

By the construction, $x \in Z(R)^{*}$ if and only if $x$ is of the form $\left(0, \overline{r_{1}}, \overline{r_{2}}, \ldots, \overline{r_{h}}\right)$. Now let $x=\left(r_{0}, \overline{r_{1}}, \overline{r_{2}}, \ldots, \overline{r_{h}}\right) \notin Z(R)$ be an element in $R$, then $x$ is invertible and indeed $x^{-1}$ is $\left(s_{0}, \overline{s_{1}}, \ldots, \overline{s_{h}}\right)$ such that $s_{0}=r_{0}^{-1}$ and $\overline{s_{i}}=-\overline{r_{i}} r_{0}^{-2}$ for $1 \leq i \leq h$. Since $|R|=\left|R^{\prime} \| U\right|=p^{(h+2) r}|Z(R)|=p^{(h+1) r}$ and $\Gamma(R)=Z(R)-\{0\}$, then $|\Gamma(R)|=p^{(h+1) r}-1$.

ii) For all $x, y \in Z(R)^{*}, x y=0$. So $\Gamma(R)$ is clearly complete.

iii) This follows from (ii).

iv) It is clear that for all $x, y \in Z(R)^{*}, d(x, y)=1$. So $\operatorname{Sup}\{d(x, y)\}=1$ for all $x, y \in \Gamma(R)$. Therefore the result is immediate.

v) When $r=1, h=0$ and $p=2$ or $3, \mathrm{t} \Gamma(R)=K_{3}$ then $n=\left(p^{(h+1) r}-1\right) \leq 2$ and hence $\Gamma(R)$ has no cycles. So, $\operatorname{gr}(\Gamma(R))=\infty$. Otherwise, for all $r, h \geq 1 n=\left(p^{(h+1) r}-1\right)>2$, so the completeness of $\Gamma(R)$ implies that $\operatorname{gr}(\Gamma(R))=2 \operatorname{diam}(\Gamma(R))+1=3$, since $\operatorname{diam}(\Gamma(R))=1$.

vi) Similar to Proposition 3.1.

Proposition 4.2 Let char $R=p$ or $p^{2}$. Then $\Gamma(R)$ is triangular, if $R$ is one of the following.

$$
\mathbf{Z}_{2} \oplus \mathbf{Z}_{2} \oplus \mathbf{Z}_{2}
$$

ii) $\quad Z_{4} \oplus Z_{2}$

iii)

$$
\mathbf{Z}_{4}[x] /\left(x^{2}+x+1\right)
$$

Proof.A zero divisor graph is triangular if $\Gamma(R)=K_{3}$. Let $R$ be a ring given by the Construction. Then $\Gamma(R)=K_{p^{h r_{-1}}}$ or $\Gamma(R)=K_{p^{(h+1) r} r_{-1}}$. It suffices to find values of $p, r$ and $h$ for which $p^{h r}-1$ or $p^{(h+1) r-1}$ equals 3. Now, let $R$ be of characteristic $p=2$. Then $\Gamma(R)=K_{3}$ when, $r=1$ and $h=2$. When $R$ is of characteristic $p^{2}$, then the graph is triangular when $p=2, r=1, h=1$ and $p=2, r=2, h=0$.

\section{Rings of characteristic $p^{k}, k \geq 3$}

Proposition 5.1 Let $\operatorname{char} R=p^{k}, k \geq 3$.

$$
\text { Then } \Gamma(R)=\left\{\begin{array}{cc}
p^{\left(\frac{k}{2}+h\right) r}-\text { partite } & \text { if kiseven } \\
p^{\left(\frac{k-1+2 h}{2}\right) r}-\text { partite } & \text { if kisodd }
\end{array}\right.
$$

Proof.Let $\lambda_{1}, \cdots, \lambda_{r} \in R^{\prime}$ with $\lambda_{1}=1$ such that $\bar{\lambda}_{1}, \cdots, \bar{\lambda}_{r} \in R^{\prime} / p R^{\prime}$ form a basis for $R^{\prime} / p R^{\prime}$ regarded as a vector space over its prime subfield $F_{p}$. Since the two cases do not overlap, we treat them in turn.

Casel : $k$ is an even integer

Let $X_{i, s}=\left\{\sum_{i=1}^{r} a_{i} \lambda_{i}+\sum_{s=1}^{h} \lambda_{s} u_{s}\right\}, 1 \leq i \leq r, a_{i} \in\left\{0, j\left(p^{\frac{k}{2}}\right)\right\}, 1 \leq j \leq p^{\frac{k}{2}}-1,1 \leq s \leq h \quad$. Then $Z(R)^{*}$ is
partitioned into the following mutually disjoint subsets, $V_{\Sigma a_{i} \lambda_{i}+\Sigma \lambda_{s} u_{s}}=X_{i, s} \backslash\{0\}, V_{1}=Z(R)^{*} \backslash \bigcup_{i, s} V_{\Sigma a_{i} \lambda_{i}+\Sigma \lambda_{s} u_{s}}$. 
These subsets are clearly nonempty and each contains nonadjacent vertices. Moreover, $Z(R)^{*}=V_{1} \bigcup \bigcup_{i, s}\left\{V_{\Sigma a_{i} \lambda_{i}+\Sigma \lambda_{s} u_{s}}\right\} \quad$. Now $\quad\left|\bigcup_{i, s}\left\{V_{\Sigma a_{i} \lambda_{i}+\Sigma \lambda_{s} u s}\right\}\right|=p^{\left(\frac{k}{2}+h\right) r}-1 \quad$ and $\left|V_{1}\right|=1 \quad$. So $\left|Z(R)^{*}\right|=p^{\left(\frac{k}{2}+h\right) r}-1$ which implies that $\Gamma(R)$ is $p^{\left(\frac{k}{2}+h\right) r}-1$ partite.

Case II: $k$ is an odd integer

Let $X_{i, s}=\left\{\sum_{i=1}^{r} a_{i} \lambda_{i}+\sum_{i=1}^{r} \sum_{s=1}^{h} \lambda_{i} u_{s}\right\}, 1 \leq i \leq r, a_{i} \in\left\{0,(j-1) p^{\frac{k+1}{2}}\right\}, 2 \leq j \leq p^{\frac{k-1}{2}}, 1 \leq s \leq h$. Then $Z(R)^{\mathrm{a}} \quad$ is partitioned into the following mutually disjoint subsets, $V_{\Sigma a_{i} \lambda_{i}+\Sigma \lambda_{s} u_{s}}=X_{i, s} \backslash\{0\}, V_{1}=Z(R)^{\mathrm{a}} \backslash \bigcup_{i, s} X_{i, s}$. The subsets are nonempty and each contains nonadjacent vertices. So $Z(R)^{*}=V_{1} \bigcup_{i, s}\left\{V_{\Sigma a_{i} \lambda_{i}+\Sigma \lambda_{i} u_{s}}\right\}$. Now $\left|\bigcup_{i, s}\left\{V_{\Sigma a_{i} \lambda_{i}+\Sigma \lambda_{s} u_{s}}\right\}\right|=p^{\left(\frac{k-1}{2}+h\right) r}-1,\left|V_{1}\right|=1$. So $\left|Z(R)^{*}\right|=\left|V_{1}\right|+\left|\bigcup_{i, s} V_{\Sigma a_{i} \lambda_{i}+\Sigma \lambda_{s} u_{s}}\right|=p^{\left(\frac{k-1+2 h}{2}\right) r}$ showing that $\Gamma(R)$ is $p^{\left(\frac{k-1+2 h}{2}\right) r}$ partite.

Corollary 5.2 Let $\operatorname{char} R=p^{k}, k \geq 3$, then

i) $\operatorname{diam}(\Gamma(R))=2$

ii) $\operatorname{gr}(\Gamma(R))=3$

$$
\text { iii) } b(\Gamma(R))=\left\{\begin{array}{l}
\frac{p^{\left(\frac{k}{2}+h\right) r}-2}{p^{(k-1+h) r}-p^{\left(\frac{k}{2}+h\right) r}}, \text { for } k \text { iseven } \\
\frac{p^{\left(\frac{k-1}{2}+h\right) r}-1}{p^{(k-1+h) r}-p^{\left(\frac{k-1}{2}+h\right) r}}, \text { for } k \text { isodd }
\end{array}\right.
$$

\section{Proof.}

i) The annihilator, $A n n_{R^{\prime} p R^{\prime}}(Z(R))=(Z(R))^{n-1} \oplus U$. So the zero divisor of the form $p^{n-1} r^{\prime}+\sum \lambda_{i} u_{i}$ is adjacent to every other nonzero zero divisor. Meanwhile, when $l+t \neq 0(\bmod \mathrm{n}$ ), $r^{\prime}, s^{\prime} \in R^{\prime}$, then zero divisors of the form $p^{l} r^{\prime}+\sum_{i=1}^{h} \lambda_{i} u_{i}$ and $p^{t} s^{\prime}+\sum_{i=1}^{h} \lambda_{i}^{\prime} u_{i}^{\prime}$ are non adjacent. Thus $\operatorname{diam}(\Gamma(R))=2$.

ii) The order of $A n n_{R^{\prime} / p R^{\prime}}(Z(R))=p^{(h+1) r}$. So every zero divisor graph of $R$ contains $K_{p^{(h+1) r}}$ subgraph. The invariants reveal that the least polygon in $\Gamma(R)$ is $K_{3}$

iii) Let $k$ be an even integer. From the definition of $V_{1}, N\left(V_{1}\right)=V_{\Sigma a_{i} \lambda_{i}}$. So $\left|N\left(V_{1}\right)\right|=p^{\left(\frac{k}{2}+h\right) r}-2$. Also $\left|V_{1}\right|=\left|Z(R)^{\mathrm{a}}\right|-\left|V_{\Sigma a_{i} \lambda_{i}+\Sigma \lambda_{s} u_{s}}\right|=p^{(k-1+h) r}-1-\left(p^{\left(\frac{k}{2}+h\right) r}-2\right)=p^{(k-1+h) r}-p^{\left(\frac{k}{2}+h\right) r}+1$. If $k$ is odd, then $\left|N\left(V_{1}\right)\right|=\left|V_{\Sigma a_{i} \lambda_{i}+\Sigma \lambda_{s} u_{s}}\right|=p^{\left(\frac{k-1}{2}+h\right) r}-1$. Also observe that $\left|V_{1}\right|=\left|Z(R)^{\stackrel{\AA}{a}}-X\right|=\left|Z(R)^{\stackrel{\AA}{a}}\right|-|X|=p^{(k-1+h) r}-1-\left(p^{\left(\frac{k-1}{2}+h\right) r}-1\right)=p^{(k-1+h) r}-p^{\left(\frac{k-1}{2}+h\right) r}$ 
. The ratio $\left|N\left(V_{1}\right)\right| /\left|V_{1}\right|$ completes the proof.

Proposition 5.3 There exists no ring in the Construction whose zero divisor graph, $\Gamma(R)$ is an $n-$ gon, $n>3$.

Proof.Suppose $R$ is not a field and the cardinality of the vertices of nonzero zero divisors, $|V(\Gamma(R))|>3$. Since $Z(R)$ is a nilpotent ideal, $\operatorname{Ann}(Z(R)) \neq(0)$. Consider $0 \neq a \in A n n(Z(R))$, then $a$ is adjacent to every other $b \in V(\Gamma(R))$. This completes the proof.

Proposition 5.4 Let $R$ be a commutative ring given by the Construction. Then $\operatorname{diam}(\Gamma(R))=0,1$ or 2 .

Proof.If $R=Z_{2} \oplus Z_{2}$, then $\left|Z(R)^{*}\right|=1$; So $\operatorname{diam}(\Gamma(R))=0$.

If $R=\mathrm{Z}_{3} \oplus \mathrm{Z}_{3}$, then $\left|Z(R)^{*}\right|=2$, so $\operatorname{diam}(\Gamma(R))=1$.

For all the other constructed commutative rings, $\operatorname{diam}(\Gamma(R))=2$ because $0 \neq a \in \operatorname{Ann}(Z(R))$ is adjacent to every other vertex in $V(Z(R))$.

Proposition 5.5 The girth $\operatorname{gr}(\Gamma(R))=\infty$ if $R$ is one of the following rings.

i) $\quad R=Z_{p}[x]\langle f(x)\rangle$, where $f(x)$ is a monic irreducible polynomial modulo $p$ of degree $r$

$$
R=Z_{2} \oplus Z_{2}
$$

iii)

$$
R=Z_{3} \oplus Z_{3} \text {. }
$$

Proof.In case (i), there exists no nonzero zero divisor.

In case (ii), there exists only one nonzero zero divisor, that is $(0,1)$.

For (iii), the ring has two adjacent nonzero zero divisors, that is $(0,1)$ and $(0,2)$. Cases (iv) and (v) are easy.

The next two results, summarize our findings on the zero divisor graphs of the equivalence classes of the rings considered in this paper.

Proposition 5.6 Let $R$ be a ring given by the Construction. Then

$\Gamma_{E}(R)=\left\{\begin{array}{cc}\frac{k}{2}-\text { partite } & \text { if } k \text { iseven } \\ \frac{k+1}{2}-\text { partite } & \text {,if kisodd }\end{array}\right.$

Case I $k$ is even.

$\Gamma_{E}(R)$ is partitioned into the following subsets

$V_{1}=\left\{(Z(R))^{l} \mid 1 \leq l \leq \frac{k}{2}\right\}$

$V_{j}=\left\{(Z(R))^{j}\right\}, \frac{k}{2}<j \leq k-1$. For each $j, V_{1} \cap V_{j}=\varnothing$ and $V_{j}$ are mutually disjoint. Moreover $V_{1} \bigcup \bigcup_{j=\frac{k}{2}}^{k-1}\left\{V_{j}\right\}=\Gamma_{E}(R)$. The result follows by counting the disjoint subsets.

Case II: $k$ is odd. 
$\Gamma_{E}(R)$ is partitioned into the following

Proof. If $k=1$ or 2 , then $\Gamma_{E}(R)$ is 1 - partite, so the result holds trivially.

For $k \geq 3$, we partition $\Gamma_{E}(R)$ into the following subsets

$$
\begin{aligned}
& V_{1}=\left\{(Z(R))^{l} \mid 1 \leq l \leq \frac{k-1}{2}\right\} \\
& V_{j}=\left\{(Z(R))^{j} \mid \frac{k-1}{2}<j<k-1\right\} .
\end{aligned}
$$

Then clearly for each $j, V_{1} \cap V_{j}=\varnothing$ and $V_{j}$ are mutually disjoint. Moreover $V_{1} \bigcup_{j=\frac{k-1}{2}}^{k-1}\left\{V_{j}\right\}=\Gamma_{E}(R)$. The result follows by counting the disjoint subsets.

Proposition 5.7 Let $R$ be a ring given by the Construction in Section 2. Then,

$$
\begin{array}{ll}
\text { i) } & \text { diameter, } \operatorname{diam}\left(\Gamma_{E}(R)\right)=2 . \\
\text { ii) } & \text { girth, } \operatorname{gr}\left(\Gamma_{E}(R)\right)=3 . \\
\text { iii) } & \text { binding number, }
\end{array}
$$

Proof.(i) and (ii) are easy to see.

$$
b\left(\Gamma_{E}(R)\right)= \begin{cases}\frac{k-4}{k} & \text { if } k \text { iseven } \\ \frac{k-5}{k-1} & \text { if } k \text { isodd }\end{cases}
$$

To prove (iii), we consider the two separate cases when $k$ is even and when $k$ is odd respectively.

If $k$ is even, $\left|V_{1}\right|=\frac{k}{2}$ while $\left|N\left(V_{1}\right)\right|=\frac{k-4}{2}$, so that $b\left(\Gamma_{E}(R)\right)=\frac{k-4}{k}$

Finally, when $k$ is odd, $\left|V_{1}\right|=\frac{k-1}{2}$ while $\left|N\left(V_{1}\right)\right|=\frac{k-5}{2}$. Then $\frac{\left|N\left(V_{1}\right)\right|}{\left|V_{1}\right|}=\frac{k-5}{k-1}$

\section{Conclusion}

In this paper, we investigated the graphical properties of the zero divisors of the rings described by the Construction in Section 2. We recommend further research on the zero divisor graphs of the rings of idealizations and their automorphisms.

\section{References}

[1] Abumoslem, A., Mohammad,E and Saeed,H. Some properties of zero divisor graphs, International Mathematical Forum, 7(16), (2012), 761-767

[2] Anderson, D.D., Naseer, M, Beck's coloring of a commutative ring, Journal of Algebra,159,(1993), 500-514.

[3] Anderson, D.F., Livingston, P.S., The zero divisor graph of a commutative ring, Journal of Algebra, 217(2), (1999), 434-447.

[4] Diestel,R., "Graph Theory", Springer- Verlag, New York, 1997.

[5] Sankeetha,S., The binding number of a zero divisor graph, International Journal of Algebra, 7(5), (2013), $229-236$. 